\title{
Pavese controcorrente: I Dialoghi con Leucò
}

\author{
ARNALDO BRUNI \\ Università degli Studi di Firenze \\ arnaldo.bruni@unifi.it
}

\begin{abstract}
Riassunto
Lo studio si propone di contestualizzare i Dialoghi con Leucò di Cesare Pavese nell'anno in cui uscirono, cioè in pieno Neorealismo (1947), senza trascurare la preistoria del dibattito sul mito, a partire dai romantici. La ricostruzione di questo filone culturale consente di intendere la difficoltà di Pavese che, nelle lettere, si sforza di giustificare la portata di questa sua prova eccentrica, opacizzata volutamente peraltro dalla pubblicazione coeva di un altro romanzo di tutt'altro segno: Il compagno. Sotto il rispetto strutturale, si sottolineano i richiami stilistici al genere del dialogo (da Platone a Leopardi) e si segnalano alcuni riusi citazionistici dedotti da Pindaro, Manzoni, Conrad e Ungaretti. Si ricorda infine l'influenza esercitata su Calvino narratore per il superamento del personaggio come tipologia obbligata e dunque si evidenzia il contributo di Pavese alla modernizzazione del romanzo nel secondo Novecento.
\end{abstract}

Parole chiave: Mito, Novecento, Neorealismo, dialogo, stile, personaggio, Pavese.

\section{Pavese against the tide : I Dialoghi con Leucò}

\begin{abstract}
The present study aims at analyzing Cesare Pavese's Dialoghi con Leucò within the context the year of its publication (1947), with Neorealism spreading all around, by taking into account the prehistory of the debate on «myth» started with romanticism. By reconstructing this cultural approach, one can understand Pavese's difficulties in trying, as it turns out from his correspondence, to clarify the meaning of this eccentric work of his; a work he even attempted to hide by publishing, in the same period of time, a totally different novel (Il compagno). Under the respect of its structure, we emphasize the stylistic similarities between the Dialoghi and the genre of dialogue (from Plato to Leopardi), and we highlight those quotations of Pindaro, Manzoni, Conrad, and Ungaretti. Finally, we recall Pavese's influence on Calvino for what concern the idea that the importance of the character withn a novel should be diminished, and, by that, we direct the attention toward Pavese's contribution to the modern conception of the novel in the second half of the 20th century.
\end{abstract}

Key words: Myth, the nineteenth-century, Neorealism, dialogue, style, character, Pavese.

Bruni, Arnaldo. 2011. Pavese controcorrente: I Dialoghi con Leucò. Cuadernos de Filología Italiana, $\mathrm{n}^{\mathrm{o}}$ extraordinario: págs. 73-82. 
Un autore che si fa critico di se stesso, si sa, non attinge necessariamente il culmine della consapevolezza. Riuscirebbe tuttavia improprio pretermettere il parere dell'interessato sulla qualità del lavoro condotto a buon fine. L'osservazione preliminare cade in taglio a proposito dei Dialoghi con Leucò, il libro che Pavese volle privilegiare per incidervi, prima del cecidere manus, un legato testamentario che è anche messaggio a futura memoria (Pavese 1990) ${ }^{1}$. Il gesto acquista valore emblematico perché si scontra con il gusto coevo, si vuol dire con l'insuccesso toccato a quell'insolita mitologia, solo in tempi recenti riscattata dal limbo dell'incomprensione corrente. Gli studiosi difatti, soprattutto negli ultimi anni, si sono impegnati a fondo nella decodifica di un libro segnato dalla discontinuità rispetto alla prassi letteraria vigente nella stagione che lo incornicia.

A segnalare l'eccentricità della suppellettile bastano del resto pochi dati riconoscibili come tipici perché diffusi nell'arco cronologico del quindicennio che distingue la parabola del Neorealismo. Il diagramma, fissato da Maria Corti fra Paesi tuoi (1941) e Metello (1955) (Corti 1978: 25-26), è distinto da alcune direttrici intese come marche caratterizzanti. In particolare, si deve registrare almeno la dimensione americana, recepita da Vittorini e Pavese, declinata insieme con la convergente lezione di Verga: «quel Verga che è in fondo a tutti i nostri sforzi» (Pavese 1966: $63)^{2}$, tanto per rammentare una battuta dell'interessato. Non sono in questione solo influenze letterarie. Si tratta piuttosto di un sommovimento di cultura che determina una linea di faglia nel quadro della tradizione, visto che le novità si avvalgono in contemporanea di un risvolto eccezionale, pensando al cinema di De Sica, Rossellini e Visconti. In un quadro così articolato e dinamico risultano dunque particolarmente incisivi quei tratti riconosciuti come determinanti perché appaiono come spie indicative di un vero e proprio ribaltamento di prospettiva. È un fatto che l'abolizione dell'io lirico, la fusione fra paesaggio e persone, l'invadenza dell'oralità nella pagina scritta, la soppressione delle descrizioni psicologiche e la prevalenza del dialogato, tanto per rammentare sommariamente gli spunti indotti da una poetica in deroga, determinano una frattura entro le misure della maniera diffusa. Lo strappo assume connotati vistosi e diviene ancora più profondo, considerando il conseguente rifiuto della forma acquisita della prosa letteraria.

Ogni movimento culturale è segnato nella sua linea di sviluppo da una costante, improntata al bioritmo della crescita e della decadenza. Il grafico grossamente abbozzato sembra culminare proprio nel 1947, l'anno topico della massima produzione neorealistica, stando almeno alle misure quantitative. Il vertice, toccato dalla spinta propulsiva della novità, è ravvisabile quindi in una stagione che allinea in serie le prove emblematiche di quella che è stata definita una «produzione torrenziale» (Corti 1978: 27). Senza pretendere di riuscire esaustivi risulta battezzato allora un lotto consistente di titoli, alcuni dei quali vanno elencati almeno come cam-

\footnotetext{
1 Si allude all'epitaffio vergato sulla prima pagina dei Dialoghi con Leucò e riprodotto fotograficamente nel controfrontespizio dell'edizione citata: «Perdono a tutti e a tutti chiedo perdono. Va bene? Non fate troppi pettegolezzi» (Pavese).

2 Si tratta di una lettera a Mario Tobino del 7 marzo 1946.
} 
pionatura provvisoria: Prologo alle tenebre di Carlo Bernari, Il cielo è rosso di Giuseppe Berto, Il sentiero dei nidi di ragno di Italo Calvino, Dentro mi è nato l'uomo di Dino del Boca, La parte difficile di Oreste Del Buono, Ė stato così di Natalia Ginzburg, L'oro di Napoli di Giuseppe Marotta, Un figlio ella disse di Silvio Micheli, Cronaca familiare e Cronaca di poveri amanti di Vasco Pratolini, Spaccanapoli di Domenico Rea.

Più che la quantità, certamente parziale, colpisce la qualità. Si tratta infatti perlopiù di opere note e in qualche modo emblematiche di protagonisti non certo di una sola stagione. Il sommario catalogo costituisce in ogni modo controprova obiettiva del gesto provocatorio di Pavese. Il quale con i Dialoghi con Leucò introduce nel tessuto compatto del modulo predominante una vera e propria scheggia antifrastica, per giunta in aperto conflitto con la tradizione antica e recente. Non si può dimenticare che lo scrittore agisca nel continuum di una tradizione. È inevitabile perciò ammettere che la possibilità di innovare scaturisca dal rapporto dialettico che si può istituire tra la prassi consolidata e le risultanze della contemporaneità. Sorprende quindi che la riflessione, pure ricca e articolata, su Leucò si sia sottratta finora a questo elementare riscontro che pure deve essere censito obbligatoriamente, almeno per sommi capi.

La gran questione della mitologia in effetti era stata liquidata nella cultura italiana, almeno in apparenza, nel 1825, con la polemica dei romantici contro il Sermone sulla mitologia di Vincenzo Monti. Il significato della querelle si ricava da un'ipotiposi attribuita a Manzoni che così si sarebbe espresso a proposito del Sermone montiano: il « $29^{\circ}$ bullettino del classicismo: come dire il bollettino di Napoleone annunciante il disastro di Mosca» (Bevilacqua 1928: 192). Il paradosso della dibattuta controversia consiste nel fatto che il Leopardi del Discorso di un italiano intorno alla poesia romantica e di Alla primavera o delle favole antiche o il Monti del Sermone, per non dire dello Schiller di Götter Griechlands, del 1788 ma tradotti in Italia nel 1822 da Giovanni Rasori, avevano da parte loro buone motivazioni per supporre che il poeta si rivolga in realtà non alla ragione, vessillifera dell' "Arido vero", ma che egli ricorra piuttosto alla «fantasia»»» con il miraggio del «portento» e della «meraviglia» (Monti 1957: vv. 90-94). La vittoriosa affermazione romantica ebbe a saldare invece, in griglia culturale egemonica, cattolicesimo e storicismo, identificando il mito con l' «idolatria» (Manzoni 1986: 320) ${ }^{3}$, in virtù della condanna dello Chateaubriand del Génie du christianisme (1802) o del Manzoni della Lettera sul romanticismo. Di qui la proposta della storia come terreno privilegiato di realizzazione di un disegno provvidenziale preordinato, secondo una prospettiva che finisce per sostenere un rinnovamento indifferibile per mezzo di premesse teoriche improprie. A questo depistaggio è lecito attribuire la responsabilità dell'isolamento della letteratura italiana da un contesto europeo diversamente articolato.

A rendere ancora più compatta la supremazia della cultura antimitologica ha contribuito in aggiunta la singolarità della scelta finale dei dioscuri polemici, cioè di bre 1823 .

${ }^{3}$ Il passo figura nella cosiddetta Lettera sul romanticismo, a Cesare Taparelli d'Azeglio del 22 settem- 
Monti e di Leopardi. I quali, dissociandosi in prima persona dai presupposti appena censiti, hanno frequentato di seguito ai loro manifesti teorici i dintorni di una letteratura alternativa, tenendo presente l'intimismo dell'ultima lirica del traduttore dell'Iliade da una parte, i grandi idilli del contino di Recanati dall'altra. L'assenza di ragioni confessionali ha consentito altrove la tenuta del modulo mitico, dalla $\mathrm{Phi}$ losophie der Mytologie (1857) di Schelling al Nietzsche di Die Geburt der Tragödie aus dem Geiste der Musik (1872), fino al Joyce dell'Ulysses (1922): o, sotto il rispetto poetico, nel trapasso che congiunge idealmente Hölderlin e Keats fino a giungere a Rilke. Negli immediati dintorni si deve citare l'esondante prevalenza del pattern, vero e proprio ritorno del rimosso, entro lo statuto del dramma moderno. Nella trafila dei recalcitranti George Steiner include una sola figura di rilievo, Bertolt Brecht (Steiner 1992: 251). Si ha tuttavia l'impressione che, nello stilare il catalogo dei resipiscenti, il critico non abbia tenuto conto a sufficienza delle vicende della cultura italiana, nell'ambito della quale avrebbe potuto trovare pane abbondante per i suoi denti. Con tutte le cautele del caso trattando di generalizzazioni sempre improprie, sembra infatti di poter azzardare che la tematica in parola sia rimasta estranea alla tradizione nostrana almeno fino alle scelte europeizzanti delle tre corone di fine secolo: il Carducci barbaro, il Pascoli dei Conviviali e il D'Annunzio alcionico. Di seguito, il modulo cade nuovamente in prescrizione, nonostante il Pirandello del teatro dei miti o qualche spunto 'magico' di Bontempelli, addirittura fino al riuso di Pavese. Il quale peraltro si giova di uno statuto teorico che sembra riagganciarsi proprio ai presupposti ottocenteschi di uno Schlegel, scrivendo in Raccontare è monotono: «Senza mito [...] non si dà poesia» (Pavese 1962: 338) ${ }^{4}$.

Tralasciando ora il merito dell'articolata fruizione storica, che andrebbe studiata a parte, i cenni sommari appena richiamati sono già sufficienti per intendere il carattere antipodico o addirittura eversivo della scelta di Pavese: il quale peraltro dimostra di avere piena consapevolezza dell'audacia implicita nell'opzione alternativa per una cultura divenuta di fronda. È agevole riconoscere in effetti nella strategia preliminare o di contorno un orientamento premonitore dei rischi a cui Pavese finiva per esporsi. Fra le cautele messe in opera rientra senz'altro la decisione di collocare il libro nella collana einaudiana dei «Saggi», quasi per cirscoscrivere l'udienza a un perimetro specialistico, laddove Il compagno vede la luce nei «Coralli». Di più, la scansione seriale del colophon (Il compagno, giugno 1947; I Dialoghi, ottobre 1947) lascia intendere che l'anticipo dell' «unico romanzo neorealista di questo scrittore» (Corti 1978: 27) giuochi un ruolo difensivo e opacizzante. La cronologia sembra assegnare alla prima tessera una funzione distrattiva, come se si trattasse di un ballon d'essai, in grado di calamitare attenzione e consenso a copertura di un'opera eccentrica ed eslege. Ancora, Il compagno pare riaffermare una fede e rassicurare il lettore ufficiale in tempi in cui le ragioni di schieramento imponevano prese di posizione inequivoche, secondo una logica schematica e radicale, tipica di un dibattito allungatosi poi ben oltre gli anni Settanta. Non è azzardato allora far dipendere dagli inconfessati presupposti apologetici la qualità non eccel-

${ }^{4}$ Schlegel (1967: 192): «Perché mitologia e poesia sono un’unità, indivisibili». 
sa del Compagno, nonostante il riconoscimento del premio Salento. La fragilità del tessuto narrativo del racconto, costruito su mitologie americane (le macchine, i camionisti, le bevute, l'insistenza sul dialogo) e pillottato di citazionismi in controtendenza, presenta, è stato osservato sagacemente, «un improbabile travestimento torinese di Jean Gabin o di Gary Cooper» (Bassani 1966: 138) ${ }^{5}$. Proprio l'inattendibilità di questo autentico 'libro dello schermo' pare assumere l'intellettualistico connotato di proporre, a fini pubblici, una sorta di segno algebrico ortodosso per equilibrare l'insostenibile eresia dei Dialoghi. I quali, in aggiunta, non potevano ricevere allora attenzione adeguata, oltreché per il contesto specifico appena rammentato, anche per la vera e propria dittatura storicistica in atto, di segno crociano e gramsciano, se è vero che ancora nel 1947 vedono la luce le Lettere dal carcere di Gramsci, nel mentre ricorre la polemica Vittorini-Togliatti con la conseguente chiusura del «Politecnico».

Di tale complessità Pavese si mostra consapevole, almeno a giudicare in base ai sondaggi degli invii editoriali, destinati a intendenti come Pietro Pancrazi, Ernesto De Martino, Santorre Debenedetti, Paolo Milano, Franco Fortini, Giuseppe Cocchiara e ad altri ancora (Pavese 1966). Si tratta di inoltri sempre accompagnati da lettere di sostegno, vòlte a precisare e a giustificare in anticipo perplessità e dubbi avvertibili perfino fra gli interlocutori più aperti. Hanno un carattere convergente ed esplicito certe dichiarazioni che, nella trama articolata del dialogo epistolare, fungono da ombrello protettivo, puntualmente avvertibile perfino, si badi bene, nella cautelosa e difensiva apertura del libro: «Potendo si sarebbe volentieri fatto a meno di tanta mitologia» (Pavese 1972: 33).

Sottolineando l'estraneità rispetto alle misure coeve, non si vuole certo negare la convergenza dell'impatto dei Dialoghi con filoni culturali emergenti che coinvolgevano la lettura ormai avviata di Frazer, di Freud e di Jung, tanto per citare l'opera di protagonisti in progressiva affermazione. A livello nazionale, i riflessi laterali sono facilmente riconoscibili perché affiorano ai margini di una trama diversamente orientata: la «Collana viola» di De Martino (Pavese-DeMartino 1991), fortemente voluta dallo stesso Pavese, o gli studi di Mario Untersteiner sulla Fisiologia del mito (1946) assumono, nella considerazione a ritroso dello storico, un ruolo pionieristico, sollecitato da altri riecheggiamenti della cultura straniera più affine, da Frobenius a Thomas Mann, da Paula Philippson a Kereny, autori questi già noti $\mathrm{o}$ in procinto di essere tradotti e scoperti in quegli anni. Il sommario catalogo propone in sostanza un filone fervido e intenso, per quanto giocato in controtendenza, che è stato ricostruito per intero (Jesi 1964; Corsini 1964: 126-30; Wlassics 1985: 127-36).

Volgendo finalmente le pagine del libro, salta all'occhio la sua singolarità strutturale. La scelta del dialogo come filo conduttore del discorso avviato, pur rifacendosi ad archetipi accusati (Platone, Luciano), si riconnette irrimediabilmente, scendendo per li rami, alle Operette morali di Leopardi. Non si deve dimenticare peraltro, a margine, che fra 1946 e il 1948 furono tradotti ben sei libri di Hemingway: del

\footnotetext{
${ }^{5}$ Si tratta di una rassegna sui Neorealisti italiani del 1948.
} 
quale, nell'anno baricentro del nostro discorso, il 1947, compaiono nella versione di Giuseppe Trevisani per Einaudi proprio i Quarantanove racconti, come dire l'opera-manifesto del dialogato in letteratura. In ogni modo, l'immanenza del pretesto leopardiano è ravvisabile nelle matrici filosofiche della scrittura o nell'atteggiamento iconoclastico che presiede all'impiego della classicità in funzione di irriverente spregiudicatezza, quale quinta dialettica oppositiva della dimensione moderna, sicché risulta calzante l'acuta definizione del libro: «Operette morali del neorealismo» (Contini 1968: 1003). La pregnanza della struttura si avverte perfino sotto il profilo tematico: perché i cosiddetti «contromiti», che accompagnano sotto forma di breve didascalia in epigrafe ventisei testi su ventisette, attivano un circuito reattivo che costituisce cornice dialettica, amplificando al quadrato la tensione discorsiva. Senza avanzare la pretesa di un'analisi esaustiva e rinviando a un pertinente studio recente (Muñiz Muñiz 1992: 99-132), non deve sfuggire intanto la divaricazione dell'ampio compasso presupposto. L'irriguardosa scorribanda prevede in sostanza l'attraversamento di tutto il mito antico come certificano i nomi dei personaggi coinvolti. Issione, Edipo e Tiresia, Ermete e Chirone, Eros e Tanatos, Achille e Patroclo, Eracle e Prometeo, e così via, disvelano dunque, secondo un'analisi recente, «Il mito» quale «racconto del nome» (Sturma 2007: 41).

La rivisitazione tuttavia acquista incisività sotto il rispetto di un'avvertita valenza autobiografica. La tematica difatti è sottoposta a una disamina che estrae spesso dal serbatoio della tradizione motivi inusitati oppure esibisce scandagli divaricati, addirittura correggendo in servizio della propria ricerca i termini della semantica arcaica. È il caso, per esempio, della diversa presentazione di Orfeo che rinuncia infine a richiamare in vita Euridice per non ricondurla ancora a ripercorrere il doloroso transito della morte. A questo aspetto si può accostare un motivo conduttore diffuso a raggiera, cioè il tema dell'incontro come occorrenza fatale e persistente, pure dichiarato concluso alla fine dei Dialoghi. Il topos in realtà, inteso come «cifra» di poesia a norma de Il mestiere di vivere ( «Fare la strada e incontrare meraviglie. Ecco il grande tema specialmente tuo»), è stato ricondotto, nella sua attiva immanenza, alla «suggestione della modernissima letteratura americana on the road» (Sturma 2007: 113). Altra prova questa del corto circuito fra archetipi e modernità innescato dalla frequentazione del mito e che si può attribuire a un vera e propria conseguenza dell' 'effetto-Vico' (Pierangeli 1995: 61-62), stando almeno al ripensamento sul filosofo antico, non per nulla riclassificato a sorpresa da Pavese come narratore nella Intervista alla radio (Pavese 1962: 295).

Un impegno di tale portata comporta di necessità una preparazione assidua, dedotta da un allenamento sistematico e stratificato. La gestazione del libro risale in effetti alla fine del 1945: è evidente però che le premesse remote si ricongiungano agli incunaboli della formazione classica dell'autore. L'intensità di tale apprendistato si misura del resto in base all'esercizio protratto delle traduzioni, in parte coeve o immediatamente successive ai Dialoghi, di tre inni omerici e della Teogonia, frutto di un'antica passione. È noto infatti, che fin dai tempi del confino di Brancaleone Calabro (1935), Pavese si era dedicato a questa formativa ginnastica, i cui risultati sono depositati nei quattro quaderni di versione da Omero e dai tragici greci conservati nel Fondo Sini-Pavese (Pavese 1981; Dughera 1982). 
Di là dalla ascendenze o convergenze letterarie, sembra utile sottolineare da ultimo l'importanza dell'adozione delle griglie formali e tematiche riconoscibili nell'architettura dei Dialoghi. Riguardo allo stile, non vi è dubbio che la singolarità dell'impianto riesca a sciogliere il nodo forse più complesso rappresentato dal caso di Pavese scrittore. A definire il quale, va sottolineato con forza il fatto che il suo esordio è di segno lirico, pensando a Lavorare stanca (Edizione di Solaria, 1936). Questa natura primaria affiora di continuo nella produzione narrativa, persino nell'opera in apparenza più eccentrica, come Paesi tuoi, dando luogo a non pochi equivoci che perlopiù indeboliscono, quando addirittura non offuscano, le prove talvolta velleitarie dello scrittore.

Ora la tematica nuova dei Dialoghi propone un terreno di sperimentazione inusitato che consente di svolgere dinamicamente i moduli originari, sospesi tra letteratura ed esigenza di referenzialità. Difatti Pavese riconosce nel mito «un midollo di realtà», nel segno di un memento vichiano in base al quale il mito è un «linguaggio» (Pavese 1972: 33) che di fatto offre il destro allo scrittore di impostare su un piano di esemplificazione globale i problemi cardinali dell'uomo contemporaneo. La lezione di Vico e degli eredi moderni impone di ripercorrere gli ampi territori della cultura classica perché il mito, come egli scrive più tardi in L'umanesimo non è una poltrona, deve essere attraversato per la «riduzione a chiarezza del mondo intero» (Pavese 1962: 282-83).

Un'operazione così ambiziosa, nata al crocevia di studi tanto diversi e complessi, dà fatalmente luogo a inevitabili contraddizioni. Chi infatti ha analizzato con competenza la difficile dialettica interna non ha mancato di rilevare un'incertezza costante fra l'esigenza di leggere nel mito una proiezione della mente umana per esorcizzare i mostri dell'inconscio oppure un primum assoluto e invalicabile (Muñiz Muñiz 1992: 114). Di più, osserva la stessa studiosa subito di seguito, Pavese sembra barcamenarsi fra la censura di un ribellismo causa di infelicità e una hýbris intesa come irrinunciabile per salvaguardare l'autonomia dell'uomo. Per giunta, egli non pare essere riuscito a decidere se stare dalla parte dei titani, simbolo della vita istintiva, o di quella degli olimpici, portatori di intelligenza apollinea.

Si deve però soggiungere, a parziale integrazione, che Pavese non aspiri ad essere iscritto nei ranghi dei filosofi professionisti. Sicché, fermo restando che le oscillazioni segnalate sono in qualche modo oggettive e dunque avvertono del carattere tragico dello statuto dell'uomo entro il cosmo, è evidente che la frequentazione del mito valga in quanto risulta personalizzata, stando a qualche suggerimento recente (Gugliemi 1967: 144).

In termini concreti, tanto per abbozzare appena un tracciato possibile, nella 'pavesizzazione' del mito non paiono trascurabili il riferimento all'ambivalenza del sesso, avvertito come maledizione (I ciechi, Le cavalle), l'insistenza sulla cultura del sangue che accompagna l'uomo come una condanna (Il fiore), il mistero inviolabile del mondo (La belva), l'idea di un tempo immobile o ciclico (La rupe). In qualche caso si riconosce l'identificazione del paesaggio come matria ( La strada) (Pavese 1972: 51, 63, 79, 104, 98), secondo una metafora che lascia intendere come Pavese proponga copertamente una relazione di contiguità sorprendente: perché la cultura delle Langhe risulta succedanea dell'archetipo arcaico. La constatazione per- 
mette di osservare che tale corrispondenza analogica ha almeno un precedente più coperto ma indubitabile in area italiana, quello di Pascoli: senza peraltro voler ora istituire una dipendenza genetica fra le esperienze di due autori in parola. Il richiamo allusivo consente di rilevare tuttavia, fatte le debite distinzioni e invocata la diversità di genere, che i Dialoghi stanno ai Paesi tuoi come i Poemi conviviali a Myricae.

Di più, sul piano stilistico, come è stato osservato (Secchieri 1991: 445-46), la proiezione mitica contribuisce a illimpidire il dettato, sospeso fra oracolarità e incisività epigrafica. Tale maniera, maculata dalla lingua witty e dalla repetitio riconosciute da altri scavi indicativi (Mutterle 1977: 37; Pierangeli 1995: 58), conferisce una dignità mai prima raggiunta al difficile e incerto equilibrio della scrittura di Pavese, bilicata, si è detto, fra spinta lirica ed esigenza comunicativa. Nella nuda, talvolta desolata, prosa di autore, scarnificata spesso di ogni aggettivazione, perfino i reimpieghi delle citazioni allusive acquistano la forza sapienziale di auctoritates assolute. Perciò il riuso di tessere espressive di Manzoni, Ungaretti, Pindaro, Con$\operatorname{rad}^{6}$ ed altri ancora lascia intravedere la grana screziata di una scrittura che si configura alla fine come una sorta di bibbia laica, in servizio di una modernità votata alla ricerca di valori pregnanti: a compenso, è necessario aggiungere sotto il rispetto sociologico, della furia distruttiva della guerra che costituisce il tragico risvolto della riflessione indotta. Proprio questa sedimentata trama culturale consente di accreditare, dalla parte del lettore, la consapevolezza del giudizio di Pavese che conferisce al libro lo spicco assoluto segnalato in apertura.

Di là dall'identikit abbozzato, più importa infine segnalare, prima del congedo, le conseguenze della spoliazione dei connotati psicologistici del personaggio come prodotto inevitabile della fruizione dei locutori mitici protagonisti dei Dialoghi. Non mi riferisco solo al contributo oggettivo del libro in servizio delle prove maggiori di Pavese, da Prima che il gallo canti a La casa in collina. Pare scontato che questi racconti si avvantaggino della prepotente spinta propulsiva che consente la definizione, in una forma rastremata e scolpita, del mescidato retroterra. Mi pare che sia il caso piuttosto di sottolineare un'altra circostanza, di solito trascurata eppure di grande momento, definibile per via indiretta attraverso le parole dell'interessato. Alludo a un passo di Pavese critico che, ragionando di altri, sembra scoprire le carte della propria poetica in elaborazione, per giunta in un intreccio cronologico accusato. Nella recensione al Sentiero dei nidi di ragno Pavese scrive: "A ventitre anni Calvino sa già che per raccontare non è necessario 'creare personaggi', bensì trasformare dei fatti in parole [...]. Ormai di scrittori che puntino sui grossi personaggi come usava una volta, non ce n'è quasi più. Cambia il mondo. Poveretto chi è rimasto coi nonni» (Pavese 1962: 273).

\footnotetext{
${ }^{6}$ Cfr. nell'ordine Pavese 1972: 43 («Passeggia brutto e testardo le campagne»: A. MANZONI, I promessi sposi, I); ivi, 44 («Tuo padre sconta la chimera»: G. UNGARETTI, Sono una creatura: «La morte / si sconta / vivendo»); ivi, 57 («Cosa sono i mortali se non ombre anzitempo?»: PINDARO, Pyt., VIII, 95); ivi, 89: «Poi viene il giorno che d'un tratto si capisce, si è dentro la morte, e da allora si è uomini fatti»: J. ConRAD, Linea d'ombra, I).
} 
Sembra evidente che Pavese, scrivendo queste parole il 16 ottobre 1947, dunque due giorni prima del 'finito di stampare' di Leucò, avesse in mente proprio i suoi dialoghi, il primo libro del momento a superare la formula del personaggio come tipologia obbligata.

Il fenomeno è così significativo da innescare una dinamica che sommuove in profondità il tessuto letterario coevo. Per averne conferma, basta pensare all'itinerario di Calvino, cioè dello scrittore che di lì a poco, lavorando su questo strappo, riuscirà ad oltrepassare la barriera del neorealismo. La successione appare inequivocabile: al Visconte dimezzato (1952) segue il Barone rampante nel 1957 che, facendo compagnia a Quer pasticciaccio brutto de via Merulana di Gadda, rende esplicita la svolta riconoscibile ormai entro le misure di quegli anni cruciali. A rendere definitivo il percorso sopraggiungerà nel 1959 il Cavaliere inesistente che sembra negare fin nel titolo il coagulo psicologico del protagonista. La prova provata della relazione supposta, peraltro inscritta nella bibliografia richiamata e nel discepolato effettivo speso da Calvino presso Pavese, si legge nella Postfazione ai Nostri antenati. L'immanenza del magistero di Pavese risulta accusata, p. es. nell'allusione ai critici che ebbero a parlare di un Calvino «favoloso»e soprattutto per la dichiarata insofferenza nei confronti della «psicologia, l'interiorità, gli interni, la famiglia, il costume, la società (specie se buona società)» (Calvino 2000: 414, 413). Di qui la predilezione confessata per personaggi che «non abbiano altro senso che la loro funzionalità nell'intreccio narrativo» (ivi, 416): spunto questo che, considerato a ritroso, risulta una definizione calzante dei protagonisti dei Dialoghi di Pavese.

Se questa ipotesi di lavoro appare plausibile, il nostro discorso può dirsi in qualche modo approdato a un esito soddisfacente. Perché alla formula vulgata, che ogni tanto capita ancora di leggere, relativa a un Pavese capofila del neorealismo italiano, va sostituita più persuasivamente la scoperta di un Pavese primo superatore del codice neorealistico nei Dialoghi con Leucò. Il che permette di chiudere questa lettura diacronica con la ricreativa conclusione di un apparente paradosso.

\section{Bibliografia}

BASSANI, Giorgio (1966): «Neorealisti italiani», in G. Bassani, Le parole preparate e altri scritti di letteratura. Torino, Einaudi.

Bevilacqua, Enrico (1928): Vincenzo Monti. La vita - l'opera - i tempi. Firenze, Le Monnier.

CAlvino, Italo (2000): «Nota 1960», in I. Calvino, I nostri antenati. Milano, Mondadori.

ContinI, Gian Franco (1968): Letteratura dell'Italia unita. Firenze, Sansoni.

CoRsinI, Eugenio (1964): «Orfeo senza Euridice: I Dialoghi con Leucò e il classicismo di Pavese», Sigma, 3/4, pp. 121-46.

CORTI, Maria (1978): Il viaggio testuale. Le ideologie e le strutture semiotiche. Torino, Einaudi.

Dughera, Attilio (1992): «Tra gli inediti di Pavese: le traduzioni dai classici greci», in A. Dughera, Tra le carte di Pavese. Roma, Bulzoni.

Guglielmi, Guido (1967): «Mito e logos in Pavese», in G. Guglielmi, Letteratura come sistema e come funzione. Torino, Einaudi, pp. 138-47. 
JESI, Furio (1964): «Cesare Pavese, il mito e la scienza del mito», in F. Jesi, Letteratura e mito. Torino, Einaudi, 1966, pp. 129-60.

MuÑIZ MuÑIZ, $M^{\text {a }}$ de las Nieves (1992): Introduzione a Pavese. Bari, Laterza.

MANZONI, Alessandro (1986): Tutte le lettere, a cura di Cesare Arieti. Con un aggiunta di lettere inedite o disperse a cura di Dante Isella, vol. I. Milano, Adelphi.

MoNTI, Vincenzo (1957): «Sermone sulla mitologia», in Vincenzo Monti, Poesie, a cura di Alfonso Bertoldi. Nuova Presentazione di Bruno Maier. Firenze, Sansoni, pp. 124-136.

MutTerle, Anco M. (1977): L'immagine arguta. Lingua, stile, retorica di Pavese. Torino, Einaudi.

PAVESE, Cesare (1962): La letteratura americana e altri saggi. Torino, Einaudi.

PAVESE, Cesare (1966), Lettere. 1945-1950, a cura di Italo Calvino. Torino, Einaudi.

PAVESE, Cesare (1972): Dialoghi con Leucò. Milano, Mondadori.

PAVESE, Cesare (1981): La Teogonia di Esiodo e tre inni omerici nella traduzione di C. P., a cura di Attilio Sughera. Torino, Einaudi.

PAVESE, Cesare (1990): Il mestiere di vivere. 1935-1950. Nuova edizione condotta sull'autografo, a cura di Marziano Guglieminetti e Laura Nay. Torino, Einaudi.

PAVESE, Cesare - De MARTINo Edoardo (1991): La collana viola. Lettere 1945-1950, a cura di Pietro Angelini. Torino, Bollati Boringhieri.

PIERANGeli, Fabio (1995): Pavese e $i$ suoi miti toccati dal destino. Per una lettura dei «Dialoghi con Leucò». Torino, Tirrenia Stampatori.

SCHLEGEL, Friedrich (1967): «Discorso sulla mitologia», in Friedrich Schlegel, Frammenti critici e scritti di estetica. Introduzione e traduzione di V. Santoli. Firenze, Sansoni.

SECCIERI, Fulvio (1991): «Il monologismo essenziale del dialogo letterario. Sui Dialoghi con Leucò di Cesare Pavese», Lingua e stile, XXVI, n. 3, pp. 429-47.

STEINER, George (1992): La morte della tragedia. Milano, Garzanti.

STURMA, Laura (2007): La parola che nomina gli dei. Saggi sulla poesia e il mito. Prefazione di Stefano Agosti. Genova, Il Melangolo.

WLASSICS, Tibor (1985): Pavese falso e vero. Vita, poetica, narrativa. Con una bibliografia della critica a cura di Luciana Giovanetti. Torino, Centro di studi piemontesi. 